Exercices Exercices de rhétorique

de rhétorique $\quad 4 \mid 2014$

Sur l'amplification

\title{
L'amplification dans la rhétorique de Louis de Grenade
}

Stéphane Macé

\section{OpenEdition}

Journals

Édition électronique

URL : http://journals.openedition.org/rhetorique/355

DOI : 10.4000/rhetorique.355

ISSN : 2270-6909

Éditeur

UGA Éditions/Université Grenoble Alpes

Édition imprimée

ISBN : 978-2-84310-292-9

\section{Référence électronique}

Stéphane Macé, «L'amplification dans la rhétorique de Louis de Grenade », Exercices de rhétorique [En ligne], 4 | 2014, mis en ligne le 28 novembre 2014, consulté le 12 septembre 2020. URL : http:// journals.openedition.org/rhetorique/355 ; DOI : https://doi.org/10.4000/rhetorique.355

Ce document a été généré automatiquement le 12 septembre 2020.

\section{(c) (1) () (2)}

Les contenus de la revue Exercices de rhétorique sont mis à disposition selon les termes de la Licence Creative Commons Attribution - Pas d'Utilisation Commerciale - Partage dans les Mêmes Conditions 4.0 International. 


\title{
L'amplification dans la rhétorique de Louis de Grenade
}

\author{
Stéphane Macé
}

\section{L'auteur, l'ouvrage}

1 Luis de Sarria, plus connu sous son surnom de Louis de Grenade (1504-1588, Luis de Granada ou Ludovicus Gratanensis), est un dominicain espagnol, auteur de nombreux ouvrages de spiritualité. Orphelin de père, il est remarqué très jeune pour sa ferveur religieuse et ses dispositions à l'art oratoire. Il se met au service des comtes de Mendoza à l'Alhambra, puis entre au couvent dominicain Santa Cruz La Real où il prononce ses vœux en 1525. Après ses études à Grenade, il rejoint en 1529 le couvent San Gregorio de Valladolid avant de devenir chapelain du duc de Medina Sidonia (à proximité de Cadix). En 1556, il connaît des difficultés avec l'Inquisition suite à la publication de son Livre de l'oraison et de la méditation (El Libro de la oración y meditación, Saragosse, 1594) et quitte l'Andalousie pour devenir provincial des dominicains du Portugal, pays dans lequel il réside jusqu'à sa mort. Directeur spirituel et confesseur de la famille royale, il refuse pourtant par humilité l'archevêché de Braga ainsi que la pourpre cardinalice. Auteur de nombreux ouvrages de doctrine religieuse, dont La Guide des Pécheurs (Guía de pecadores, 1556) et le Mémorial de la vie chrétienne (Memorial de la vida cristiana, 1561), il développe une pensée fortement influencée par le thomisme et par la mystique de Jean d'Avila dont il retient aussi l'attachement à l'idéal cicéronien du style.

2 Le traité La Rhétorique de l'Église, rédigé en latin, fut publié en 1576 à Lisbonne (Ecclesiasticae rhetoricae, sive de ratione concionandi libri sex, Olysippone, Ant. Riberius ${ }^{1}$ ). Le texte latin connaît ensuite plusieurs éditions à l'étranger : Cologne, Birckmann, 1578 et 1594 ; Venise, Francisco Ziletto, 1578 ; Cologne, A. Quentelius, 1611; Paris, Guillaume Pelé, 1635 - plusieurs éditions numérisées sont disponibles via le KVK ${ }^{3}$. Le texte que nous proposons est celui de la traduction française de Nicolas-Joseph Binet (Paris, Jean Villette, 1698) : Binet était recteur de l'université et nous a également laissé une anthologie de sermons de Louis de Grenade, parue la même année à Paris chez 
Claude Hérissant. Le privilège concerne aussi bien la traduction des sermons que celle de la Rhétorique de l'église (qui serait, comme l'indique explicitement le titre, traduite à partir du texte espagnol plutôt que du latin ${ }^{4}$ ) ; il a été accordé en 1694, mais suite au décès de Claude Hérissant, il a été transféré en 1698 à Jean de Nully, qui a choisi de le partager avec les libraires Pierre Hérissant, Jean Villette et Louis Roulland. En 1698 paraissent donc simultanément deux éditions, chez Jean Villette (cote BNF : D-15268; cote Arsenal: $8 \mathrm{~T} 6079$; et sur Google Livres) et chez Pierre Hérissant (cote BNF : D-36684 et Microfilm M 1098) ${ }^{5}$.

\section{Les développements sur l'amplification : principes et enjeux}

Le texte de Louis de Grenade présente un double intérêt : parmi tous les ouvrages que nous avons pu consulter, il est celui qui fait la plus large place à l'amplification et il mobilise conjointement de nombreuses sources théoriques (Quintilien, Cicéron, Érasme...); en outre, l'auteur propose une large palette d'exemples (principalement empruntés aux Pères de l'Église et à la littérature spirituelle ${ }^{6}$ ) pour illustrer son propos : la démonstration est donc d'une très grande clarté.

4 De nombreux théoriciens de l'âge classique semblent gênés par l'absence de développement autonome chez Aristote ou Cicéron (Quintilien est le premier à consacrer un chapitre spécifique à l'amplification). Ils se contentent souvent de chapitres assez brefs ou éludent même la question, comme le fait par exemple René Bary :

Je ne donne point d'exemple de l'amplification, parce qu'elle souffre l'amas des définitions, la multitude des dépendances, la comparaison des inégaux, la rencontre des contraires, les gradations, les derivez, les distributions, les Prosopopées, \& qu'il faudroit faire une espece de livre, pour donner l'usage de cette figure ${ }^{7}$.

D'autres encore cantonnent l'étude de l'amplification aux « exercices préparatoires ", à côté de la narration, de la chrie ou de la pratique de la sentence. Un tel choix n'est pas sans intérêt puisqu'il permet d'insister sur la dimension fortement technique de la notion (que notre époque a sans doute tendance à minimiser): privilégiant une approche concrète, les conseils pratiques que l'on peut dispenser à un élève permettent de comprendre « de l'intérieur » le fonctionnement de l'amplification et de bien saisir à quoi correspondent les différentes sous-catégories envisagées. Toutefois, cela conduit aussi à marginaliser le procédé, voire à déprécier quelque peu son statut dans la mesure où les "exercices préparatoires " sont systématiquement présentés en annexe des traités théoriques, en complément du propos général et selon une perspective scolaire nettement plus marquée.

6 À l'inverse, Louis de Grenade choisit d'intégrer l'étude de l'amplification à sa théorie générale de l'art oratoire, allant jusqu'à consacrer au procédé un livre entier (livre III, p. 213-336). La lecture de l'auteur, particulièrement extensive, englobe un certain nombre d'éléments plus habituellement abordés dans un autre contexte: la description, l'analyse de figures comme la sermocinatio ou la prosopopée, l'étude des procédés servant à exprimer les passions. Il peut être intéressant de se reporter à la table générale de l'ouvrage pour bien comprendre la logique de l'auteur.

7 Le premier livre (dépourvu de titre) propose une définition générale de la rhétorique et insiste longuement sur l'intérêt que les prédicateurs peuvent trouver à employer les 
ressources de l'art oratoire : il s'agit de montrer que cette discipline, quoique issue du paganisme, est pleinement compatible avec la religion chrétienne et que l'homme d'Église est à sa manière une incarnation parfaite du vir bonus dicendi peritus cher à Quintilien ${ }^{8}$. Pour reprendre le titre du chapitre 5, «l'observance des Regles de l'Eloquence n'empêche point de suivre dans ses discours les mouvemens \& les impressions de l'Esprit de Dieu ».

8 Après quelques éléments de définition générale (essentiellement l'exposé des cinq parties de l'art oratoire), le second livre - également dépourvu de sous-titre - aborde rapidement les différents états de cause, la question des lieux et, de façon beaucoup plus détaillée, les différents mécanismes logiques qui soutiennent le système de la preuve: induction / déduction, syllogismes, enthymèmes, dilemmes, sorites, raisonnement par énumération, etc. La fin de ce livre II aborde aussi rapidement les passions et la façon dont on doit les « répandre » dans le raisonnement oratoire, ainsi que les «ornements» que constituent la sentence ou l'épiphonème, explicitement désignés comme des moyens d'amplifier le discours. On peut voir là une forme de transition vers la troisième partie, spécifiquement dévolue à l'amplification, et dont nous reproduisons ici le sommaire :

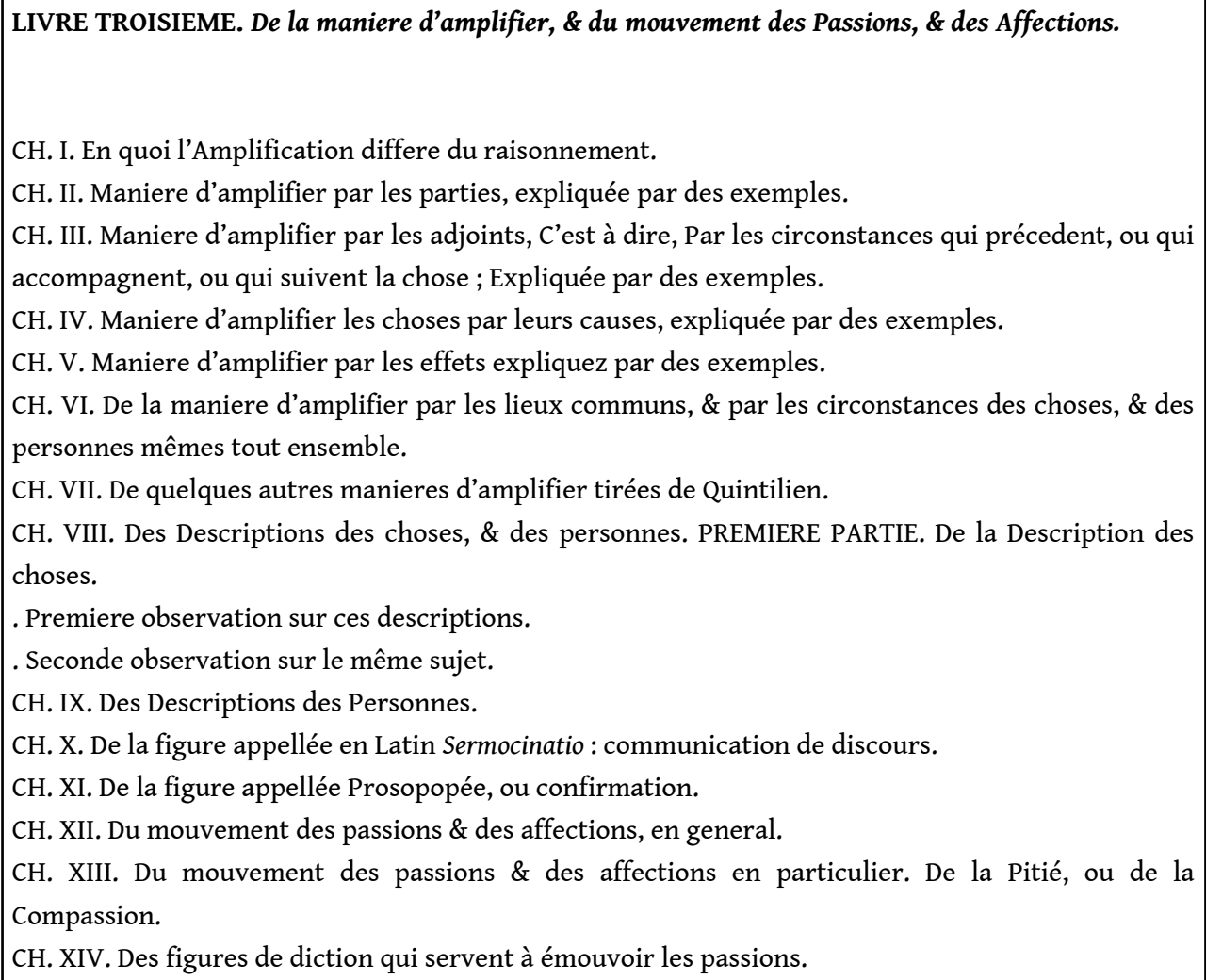

9 Les sept premiers chapitres reprennent en détail les catégories présentées par Quintilien dans la section VIII, 4 de l'Institution oratoire, mais avec des déplacements d'accents sensibles. Le premier chapitre étudie les rapports, assez complexes, entre l'amplification et le système de la preuve; les quatre suivants, 2 à 5 , développent à grand renfort d'exemples issus de la littérature religieuse (les livres des Prophètes dans la Bible, saint Paul, saint Cyprien et les Pères de l'Église, Lactance...) la catégorie de 
l'amplification par accumulation (per congeriem), en détaillant les principales sousespèces identifiées par le modèle latin (accumulation des parties, des adjoints, des causes ou des effets). Mais Quintilien est croisé ici avec une autre source majeure, le De copia verborum (1512) d'Érasme auquel sont empruntés plusieurs exemples. Le sixième chapitre s'apparente à une synthèse illustrant une utilisation mixte des précédentes techniques. Enfin, le septième chapitre fait retour de façon assez fidèle au chapitre VIII, 4 de Quintilien, explicitement désigné comme le principal modèle de l'auteur, et dont il est en grande partie un décalque direct. Certains passages de Quintilien sont toutefois omis, et Louis de Grenade ajoute à l'inverse un certain nombre d'exemples issus de la littérature religieuse.

Tout se passe donc comme si Louis de Grenade, influencé par Érasme et la théorie humaniste de la copia, avait voulu donner une place plus importante à l'amplification par accumulation, au détriment peut-être des autres catégories inventoriées par Quintilien (amplification par gradation, par comparaison, par raisonnement, ou encore l'amplificatio verborum et l'emphase) : le choix de présenter ces chapitres à part, avant même le résumé de la théorie de Quintilien, et de les illustrer par de nombreux exemples n'est assurément pas un geste neutre. On perçoit bien ici quelles peuvent être les ambitions de l'auteur, dont la démarche est typique de l'esprit syncrétique des humanistes de la Renaissance et dont l'érudition sans faille est aussi tout à fait conforme à ce que l'on peut attendre d'un adepte de la règle de saint Dominique. Audelà du travail de compilation s'exerce ici une pensée véritablement audacieuse et ingénieuse qui n'entend renoncer à aucune de ses prérogatives.

11 La suite du livre III - chapitres 8 à 14 - manifeste cette même liberté et s'écarte délibérément de la progression suivie par Quintilien. Celui-ci, après le chapitre VIII, 4 de son Institution oratoire, passe à tout autre chose (les différents types de sentences, l'étude des tropes, puis, avec le Livre IX, l'examen systématique des figures), alors que Louis de Grenade prend le parti d'intégrer tout un ensemble d'éléments nouveaux à la réflexion sur l'amplification. Du fait de ce choix audacieux, le propos des chapitres 8 à 14 a souvent tendance à s'écarter assez sensiblement de la problématique d'ensemble, même si l'auteur, de temps à autre, prend bien soin de rappeler l'orientation générale du livre III en soulignant, par des formules brèves et synthétiques, l'articulation entre les notions étudiées et l'amplification.

Sont ainsi successivement abordées la description (et en particulier l'hypotypose et sa dimension fortement pathétique), des figures comme la sermocination, la communication ou la prosopopée (qui toutes supposent une utilisation singulière de la voix et une forme de relation particulière du discours à autrui), et enfin les passions - dont on connaît l'importance, depuis la Rhétorique d'Aristote, dans le champ des études rhétoriques : annexer ce point à la théorie de l'amplification est assurément une modification majeure. Il s'agit moins à proprement parler de sous-catégories de l'amplification que de connexions naturelles que l'on peut établir entre cette notion et différents éléments de l'art oratoire. Notons que la présentation des subdivisions et des sous-titres n'est pas extrêmement rigoureuse (dans la table comme dans le texte, aucune «deuxième partie " n'apparaît après la "première partie » dévolue à la " description des choses »), mais la structure n'en est pas moins transparente.

L'amplification, dans ce troisième livre de La Rhétorique de l'Église, est abordée comme une ressource de l'inventio. La toute première phrase du chapitre liminaire suffit à lever toute ambiguïté à ce sujet: «Il est constant que l'Amplification fait une partie de 
l'invention [Amplificationem, quam inventionis partem esse constat], ou de la maniere de trouver des preuves \& des argumens. " C'est là un traitement assez classique avant Quintilien, mais un peu surprenant pour un auteur qui prend aussi explicitement pour modèle l'Institution oratoire. En effet, les livres VIII et IX de Quintilien sont explicitement consacrés à l'elocutio ${ }^{9}$. Certes, on peut montrer que Quintilien ne bascule pas totalement du côté de l'elocutio, mais le fait de placer le chapitre consacré à l'amplification au milieu du livre VIII marque chez lui un glissement très perceptible dans cette direction.

Chez Louis de Grenade, l'ordre canonique des "parties de l'art oratoire » est respecté tout aussi méthodiquement que chez Quintilien. La Rhétorique de l'église consacre son livre suivant (IV) à la dispositio, appliquée au genre religieux du sermon et à ses différentes variantes. On comprend donc rétrospectivement que les deuxième et troisième livres (preuve et amplification) formaient une unité, dévolue à l'inventio. Le cinquième livre, sans surprise, aborde alors l'elocutio et comprend un répertoire extrêmement complet des figures de style, ainsi qu'un exposé sur les différents niveaux de style et la bonne pratique de l'ornement. La memoria n'est pas abordée par le traité, qui consacre le sixième et dernier livre à l'actio - en restreignant naturellement le champ d'application à la pratique religieuse de la prédication. Louis de Grenade se rattache donc plus nettement à la tradition antérieure à Quintilien, celle qu'illustrent notamment Aristote, ou, chez les Latins, la Rhétorique à Herennius (II, 47-50 ou IV, 11-12) qui relie directement l'amplification à la question des lieux.

\section{NOTES}

1. Rouen, BM, Fonds Cas, cote 0407 ; Châlons-en-Champagne, BM, fonds Garinet, cote GT17787.

2. En trois livres seulement, et sous la signature d'Agostino Valiero, évêque de Vérone (1531-1606), avec une intéressante préface expliquant tout le mérite mais aussi la difficulté pour les simples prêtres de l'ouvrage de Louis de Grenade (l'ouvrage est dédié à Charles Borromée ; sur Google Livres).

3. Dernière traduction en espagnol (avec en regard le texte latin), par Manuel López-Muñoz : Fray Luis de Granada : los seis libros de la "Retórica Eclesiástica", o método de predicar, Logroño, Instituto de Estudios Riojanos, 2010. Le texte latin, lui, est numérisé en mode texte sur le site www.cvmas.org.

4. La Rhétorique de l'Eglise, ou L'Eloquence des predicateurs. [...] Et Traduite nouvellement de l'Espagnol en François.

5. Il existe un abrégé de la Rhétorique de l'Eglise [...] par le R. P. Pierre de Rians, Avignon, Fr. Girard, 1743 (Google Livres) et une réédition de 1829 de la traduction Binet: Rhétorique ecclésiastique ou Traité de l'éloquence des prédicateurs [...] Nouvelle édition, revue et considérablement retouchée par un professeur de théologie, Lyon, Fr. Guyot, 1829, deux tomes (Gallica; t. I sur Google Livres). Le livre III est dans le tome I, p. 239-370; à la fin du tome II, p. 421-448, se trouve une "Analyse oratoire de deux discours de Saint Pierre rapportés dans les chapitres II et III des Actes des Apôtres ", où le théologien de 1829 applique les principes de Grenade, ou plutôt, en l'occurrence, de Jean Chrysostome. L'ouvrage met en épigraphe une formule de François de Sales (ép. 47) : «Que Grenade soit votre second Bréviaire. »

6. Nous remercions vivement Benoît Gain pour les références de patristique grecque. 
7. René Bary, La Rhetorique Françoise, Paris, Pierre Le Petit, 1665, p. 415.

8. «Un homme de bien habile à parler ", Institution oratoire, XII, I, 1 (trad. Jean Cousin, Paris, Les Belles Lettres, 1980) : définition explicitement reprise à Caton l'Ancien.

9. « Nous allons traiter désormais de la théorie du style [elocutionis rationem] », Institution oratoire, VIII, pr., 13 ; « Donc, ce que les Grecs appellent phrasis nous le nommons en latin elocutio », VIII, I, 1, toute première phrase (trad. Jean Cousin, Paris, Les Belles Lettres, 1978).

\section{AUTEUR}

\section{STÉPHANE MACÉ}

Univ. Grenoble-Alpes - RARE Rhétorique de l'Antiquité à la Révolution 